$\operatorname{sein}^{35}$. In der zweiten Jahreshälfte 1992 sollen gegen zwei weitere Verdächtige in Adelaide bzw. vor einem bislang noch nicht benannten Gericht im Bundesstaat Queensland die Verfahren eröffnet werden ${ }^{36}$. Ob ein letzter fünfter Fall jemals vor Gericht kommen wird, ist zur Zeit noch unklar ${ }^{37}$.

\title{
Uwe Günther \\ Das Ende einer Fraktion - Eine Reise durch juristisches Niemandsland
}

Am 3. 1 2. 1990 scheiterte die Partei DIE GRÜNEN (West) im Wahlgebiet der alten Bundesländer an der $5 \%$-Hürde des Wahlgesetzes. Mit der Konstituierung des neuen Bundestages am 20.12. 1990 hörte die Bundestagsfraktion DIE GRÜNEN/BÜNDNIS 90 auf, zu bestehen. ${ }^{I}$ In den Bundestag zog die Gruppe BÜNDNIS 90/GRÜNE ein, deren Abgeordnete im Wahlgebiet der neuen Bundesländer gewählt worden waren und die sich nicht als Rechtsnachfolgerin der Fraktion DIE GRÜNEN/BÜNDNIS 90 verstand.

Das politische Ende der Bundestagsfraktion DIE GRÜNEN/BÜNDNIS 90 war nicht identisch mit ihrem verwaltungsrechtlichen Schicksal: Am 20. I2. 1990 verfügte die Fraktion in Abwicklung über ca. 4,s Mill. DM bzw. - nach Abzug von Verbindlichkeiten - über 2,5 Mill. DM, ca. I25 Mitarbeiter und Mitarbeiterinnen, ca. 125 Abgeordnetenmitarbeiter und -mitarbeiterinnen und über Gerätschaften im Anschaffungswert von ca. I,5 Mill. DM. Ferner existierten diverse langfristige Verpflichtungen aus Wartungs-, Leih- und Lieferungsverträgen. Zu beantworten war die Frage, welche arbeitsrechtlichen, sozialrechtlichen, verwaltungsrechtlichen und zivilrechtlichen Konsequenzen aus dem politischen Ende der Fraktion zu ziehen waren. $^{2}$

Ein Fraktionsgesetz, aus dem sich rechtliche Konsequenzen aus dem politischen Ende der Fraktion hätten ablesen lassen, gab und gibt es nicht. ${ }^{3}$ In der juristischen Literatur wird der Rechtscharakter einer Fraktion höchst unterschiedlich beurteilt, und demzufolge sind die rechtlichen Konsequenzen, die aus der rechtlichen Charakterisierung einer Fraktion gezogen werden, unterschiedlich. ${ }^{4}$ In der Vergangenheit hat es zwar bereits Fraktionen gegeben, die infolge der Sperrklausel nicht wieder in

35 Sydney Morning Herald, is.6., 16.6. und 23.6.1991; Verfahrenseinstellung mangels Bewerse am 29. 7. 1992 .

36 Sydney Morning Herald, is.6. und 16.6.1992; Manuskript-Schluß: September 1992.

37 Prof. Dr. Konrad Kwiet (Fn. 19), in einem Gesprach Ende Juni 1992.

I Die Fraktion DIE GRUंNEN hatte sıch am 3. 10.1990 mit der Volkskammerfraktion BUNDNIS $90 \mathrm{zu}$ einer Fraktion zusammengeschlossen unter dem Namen "Fraktion DIE GRUंNEN/BUंNDNIS 90«.

2 Durch Beschluß des Fraktionsvorstandes wurde der Autor dieses Beitrages, der ab Mai 1990 kommissarisch als Fraktionsgeschaftsfuhrer der Bundestagsfraktion tatig war, zum Geschaftsfuhrer der Fraktion in Liquidation bestellt und damit mit der Abwicklung betraut.

3 Zur Zeit gibt es im Bundestag und in verschiedenen Bundeslandern Überlegungen zu einem »Fraktionsrechtsstellungs-Gesetz «. In Kraft getreten ist das Bayerische Fraktionsgesetz vom 26. 3. 1992

4 Vgl. den Überblick bei Bick, Die Ratsfraktion, 1989, S. 49 ff.; Jekewitz, Polıtusche Bedeutung, Rechtsstellung und Verfahren der Bundestagsfraktion, in: Parlamentsrecht und Parlamentspraxis, Hrsg. Schneider/Zeh, 1989, S. 1021 (S. 1045 ff.).

Kretschmer, Fraktionen, in: Handschuh/Kremer/Kretschmer/Zeh, Wegweiser Parlament, 1987, S. 253, S. $283 \mathrm{ff}$. 
den Bundestag bzw. in die Landtage eingezogen sind. Ihnen standen allerdings le-

diglich geringe finanzielle Volumina zur Verfügung, und daher war die Bandbreite der Probleme erheblich kleiner's Rechtsprechung, die die unterschiedlichen Literaturauffassungen hätte bündeln können, gibt es wenig. ${ }^{6}$

\section{Die Vorgeschichte}

Um die arbeits- und sozialrechtlichen Probleme der Abwicklung zu verstehen, ist es erforderlich, zuvor die arbeitsrechtlichen Besonderheiten, die bei Mitarbeitern und Mitarbeiterinnen einer Fraktion bzw. Abgeordneten bestehen, zu erklären:

Fraktionsmitarbeiter erhalten in der Regel unbefristete Arbeitsverträge, wobei arbeitsvertraglich auf den BAT Bezug genommen wird. Ihre Bezahlung erfolgt durch die Fraktion bzw. die Fraktionszuschüsse. Abgeordnetenmitarbeiter werden befristet bis zum Ende der Legislaturperiode bzw. bis zum Ausscheiden des Abgeordneten aus dem Bundestag eingestellt. Die Höhe ihrer Bezahlung richtet sich nicht nach dem BAT, sondern wird durch »Ausführungsbestimmungen« des Ältestenrates geregelt. Jedem Abgeordneten steht in gleicher Höhe eine "Mitarbeiterpauschale" zu. 7 Die Mittel werden von den Abgeordneten nicht selbst verwaltet, sondern von der Bundestagsverwaltung zur Auszahlung gebracht. Die arbeitsrechtlich unterschiedlichen Möglichkeiten zur Ausgestaltung des Arbeitsverhältnisses führen regelmäßig dazu, daß Abgeordnetenmitarbeiter schlechter als Fraktionsmitarbeiter behandelt werden. Der deutlich unterschiedlichen Behandlung entspricht in der Realität eine undeutliche Unterscheidung der Funktionen. Um die arbeitsrechtliche Ungleichbehandlung von Fraktionsmitarbeitern und Abgeordnetenmitarbeitern auszugleichen, hatten sich die Abgeordneten der Fraktion DIE GRÜNEN freiwillig bereit erklärt, die "Mitarbeiterpauschale» in einen gemeinsamen "Pool « zu geben, aus dem alle Abgeordnetenmitarbeiter bezahlt wurden. Dieser wurde faktisch von der Fraktionsgeschäftsführung verwaltet, wie diese auch faktisch Arbeitgeber der Fraktionsmitarbeiter und -mitarbeiterinnen war. Durch die skizzierte »Pool-Bindung und einen einheitlichen Arbeitgeber wurde Gleichbehandlung realisiert, allerdings bloß faktisch und nicht juristisch.

Die juristische Grenze der Gleichbehandlung zeigte sich insbesondere bei der Auseinandersetzung um die Verabschiedung eines Sozialplanes im Herbst 1990: In dieser Zeit wurde fraktionsintern über die Konsequenzen der näher rückenden Bundestagswahl nachgedacht. Klar war allen, daß es - wie auch beim vorherigen Legislaturperiodenwechsel-.zu Kündigungen kommen würde. ${ }^{8}$ Ein Sozialplan sollte daher die prognostizierten Kündigungen abfedern. Um den Inhalt des Sozialplans kam es $\mathrm{zu}$ heftigen Meinungsverschiedenheiten, die auch in einem Streik ihren Ausdruck

5 Vgl. zur Entwicklung der Fraktionszuschusse v. Arnım, Staatlıche Fraktionsfinanzierung ohne Kontrolle, 1987, S. 66.

Die FDP war verschiedentlich gezwungen, Fraktionen auf Landerebene abzuwickeln. Zu rechtlichen Auseinandersetzungen kam es meines Wissens nicht. Das mag mit dem geringen finanziellen Volumen und der geringen Anzahl von beschäftigten Personen zusammenhängen, aber auch damit, daß es für die FDP leichter sein durfte als fur DIE GRUNEN, Personal bei »befreundeten « Instituten und Unternehmungen unterzubringen.

196I gelangte die Deutsche Partei nicht mehr in den Bundestag. Die Abwicklungsprobleme der Deutschen Partei mußten vergleichsweise klein gewesen sein, da 1961 alle Fraktionen des Bundestages insgesamt nur 2 Mill. DM erhielten, also weniger, als die Fraktion DIE GRUNEN/BUंNDNIS 90 zur Liquidation zur Verfügung hatte.

$6 \mathrm{Vgl}$. etwa BVerwG NJW 1985 , S. 2344.

7 Gemaß $\$ I 2 Abs. 3 des Abgeordnetengesetzes. Die Mitarbeiterpauschale betragt z. Zt. 12 960,-DM.

8 Wenngleich die wenigsten damit rechneten, daß DIE GRÜNEN den Einzug in den Bundestag nicht schaffen wuirden. 
fanden. Der Grund lag letztlich in den haushaltsrechtlichen Besonderheiten der Fraktionszuschüsse einerseits und der Mitarbeiterpauschale andererseits. Während die Fraktion aus den Fraktionszuschüssen Rücklagen bilden konnte ${ }^{9}$ und dies auch tat, konnten Abgeordnete über die Mitarbeiterpauschale nicht frei verfügen, sondern sie nur zur Bezahlung von Mitarbeitern und Mitarbeiterinnen verwenden, nicht aber zur »Rücklagenbildung « ${ }^{10}$. Während mithin Rücklagen aus Fraktionszuschüssen für einen Sozialplan der Fraktionsmitarbeiter zur Verfügung standen, gab es solche Rücklagen für Abgeordnetenmitarbeiter nicht. Die gewollte Gleichbehandlung zwischen beiden Gruppen drohte also gerade im Konfliktfall, für den sie greifen sollte, zu scheitern. In dieser Situation wurde ein Kompromiß durch mehrere Maßnahmen erreicht:

Die Abgeordneten beschlossen, einen "Spendentopf « zu bilden, der zur Finanzierung cines Sozialplans für Abgeordnetenmitarbeiter dienen sollte. Als klar war, daß der "Spendentopf « nicht annähernd das Volumen erreichte, das notwendig zur Finanzierung eines Sozialplans war, der den Vorstellungen der Abgeordnetenmitarbeiter und -mitarbeiterinnen entsprach, und die fraktionsinternen Auseinandersetzungen hohen Unterhaltungswert in der Öffentlichkeit bekamen, leistete der Bundesvorstand der Partei DIE GRÜNEN eine Ausfallbürgschaft und verpflichtete sich, die Differenz zu bezahlen zwischen dem Betrag, den die Abgeordneten lt. Beschluß hätten spenden sollen, und dem Betrag, den sie gespendet hatten. Zum dritten wurde vereinbart, daß - soweit Spenden und Ausfallbürgschaft nicht ausreichen sollten eine Abfindung statt in »Geld « in "Lohnfortzahlung ohne Beschäftigungspflicht « durch zeitweilige Anstellung bei dem »Pool«, der auch in der neuen Legislaturperiode vorgesehen war, bewirkt werden sollte. Der durch Spenden, Ausfallbürgschaft und des vereinbarten Prinzips »Lohnfortzahlung anstelle von Geld « zustandegekommene Kompromiß im Sozialplan war rechtlich von vornherein fragil, weil er auf der unterstellten Zustimmung der neuen Fraktion beruhte. Diese Fragilität kam nach dem 3. I 2. I 990 zum Tragen.

\section{Fraktionsmitarbeiter und Fraktionsmitarbeiterinnen}

Nach dem 3. 1 2. 1990 standen der Fraktion in Abwicklung Rücklagen aus Fraktionszuschüssen in Höhe yon 2,5 Mill. DM zur Verfügung. Die Frage war jedoch, ob die Rücklagen ausschließlich zur Finanzierung des Sozialplans verwendet werden durften. $\mathrm{Zu}$ bedenken war, ob gegenüber den Mitarbeitern und Mitarbeiterinnen die Kündigungsfristen der Arbeitsverträge eingehalten werden mußten. War eine Kündigung notwendig? Konnte zurückgegriffen werden auf den juristischen Grundsatz des Wegfalls der Geschäftsgrundlage? Folgt das Arbeitsrecht dem Parlamentsrecht und konnte die Bezahlung der Mitarbeiter und Mitarbeiterinnen mit Erlöschen der Fraktion eingestellt werden? ${ }^{11}$ Im Interesse einer schnellen Abwicklung und im Interesse der Mitarbeiter und Mitarbeiterinnen wäre es sinnvoll gewesen, mit dem Ende der Fraktion auch die Arbeitsverhältnisse enden zu lassen. In diesem Falle hätte den Mitarbeitern und Mitarbeiterinnen die Abfindung gemäß Sozialplan in voller Höhe ausgezahlt werden können, sie hätten sich sofort arbeitslos melden und sofort

9 Was der Bundesrechnungshof fur nicht zulassig hảlt, vgl. die unveroffentlichte Problemskızze des Bundesrechnungshofes vom 24.4. 990 .

Io Die entsprechenden Regelungen des Abgeordnetengesetzes suchen zu verhindern, daß Gelder privaten Kanalen von Abgeordneten zufließen.

I I Bick, (Fn. 4), S. I91, und Kretschmer, (Fn. 4), S. 203, vertreten den Standpunkt, daß Arbeitsverhaltnisse mit einer Fraktion unter der auflosenden Bedingung geschlossen werden, daß der Arbeitgeber "Fraktion « fortbesteht. 
Arbeitslosengeld beziehen können. Überdies bedeutet die Einhaltung der Kündigungsfristen Lohnfortzahlung für Mitarbeiter und Mitarbeiterinnen im Kündigungszeitraum und damit, daß Personal ausschließlich für Zwecke der Lohnbuchhaltung vorgehalten und bezahlt werden mußte.

In Verhandlungen mit dem Arbeitsamt Bonn und dem Landesarbeitsamt in Düsseldorf wurde versucht, den Rechtsstandpunkt durchzusetzen, daß mit dem politischen Ende einer Fraktion auch die Arbeitsverhältnisse enden. Die Arbeitsverwaltung folgte diesem Rechtsstandpunkt nicht. Sie sah rechtlich die Fraktion in keiner anderen Situation als ein gewerbliches Unternehmen. Da die Fraktion noch über Geldmittel verfügte, mußten diese nach Auffassung der Arbeitsverwaltung verwandt werden, um Mitarbeiter und Mitarbeiterinnen während der Laufzeit der ordentlichen Kündigungsfrist zu bezahlen. Die Arbeitsverwaltung kündigte an, daß diejenigen Arbeitnehmer und Arbeitnehmerinnen, die eine Abfindung erhalten würden und die sich ohne Einhaltung der Kündigungsfrist arbeitslos melden würden, mit einer Sperrzeit belegt werden würden. Im Interesse einer schnellen Klärung der Angelegenheit blieb nichts anderes übrig, als sich den Rechtsstandpunkt der Arbeitsverwaltung zu eigen zu machen und allen Mitarbeitern und Mitarbeiterinnen ordentlich zu kündigen unter Freistellung von der Arbeit und unter Fortbezahlung der Bezüge. Den Rechtsstandpunkt der Arbeitsverwaltung nicht zu akzeptieren hätte bedeutet, sich auf langwierige Prozesse mit ungewissem Ausgang einzulassen. Gleichwohl bleiben Zweifel wegen des angenommenen Rechtsstandpunktes. Was wäre gewesen, wenn die Fraktion in Anerkennung des Rechtsstandspunktes des Bundesrechnungshofes keine Rücklagen gebildet hätte? Hätte auch in diesem Fall den Mitarbeitern ordentlich gekündigt und Konkurs angemeldet werden müssen? Kann eine Institution des Verfassungsrechts in Konkurs gehen?

\section{Abgeordnetenmitarbeiter und Abgeordnetenmitarbeiterinnen}

Die Fraktionsmitarbeiter waren dadurch, daß ihnen gegenüber Kündigungsfristen galten und sie freigestellt waren, gegenüber Abgeordnetenmitarbeitern sozial erheblich besser gestellt: Diese erhielten Gehalt bis zum 31.12. 1990 und mußten sich ab dem I. 1. 1991 arbeitslos melden. Die Sozialplanregelungen, die zu ihrem Schutz und im Interesse der Gleichbehandlung gedacht waren, blieben Papier, weil einerseits der "Spendentopf " zu schmal ausgestattet war, und andererseits die als Surrogat für fehlendes Geld gedachte Regelung "Lohnfortzahlung bei Freistellung « nicht greifen konnte infolge des Fehlens des entsprechenden Arbeitgebers Bundestagsfraktion.

\section{Schwangere und Schwerbehinderte}

Die Fraktion beschäftigte zu Beginn der Abwicklung sieben Schwerbehinderte, zwei Schwangere und zwei Frauen, die im Erziehungsurlaub waren. Unter Zugrundelegung des Standpunktes der Arbeitsverwaltung konnte ihnen gemäß Mutterschutzgesetz bzw. Schwerbehindertengesetz nicht gekündigt werden. Hierzu war die $\mathrm{Zu}$ stimmung der Hauptfürsorgestelle bzw. des Landschaftsverbandes notwendig. Das Zustimmungsverfahren bewirkte, daß sich die ordentliche Kündigungsfrist der Betroffenen verlängerte. Letztlich wurde in allen Fällen die Zustimmung erteilt. Das Zustimmungsverfahren ist in Bezug auf die Liquidation einer Fraktion insofern eigenartig, als ein solches Verfahren unterstellt, die Zustimmung könne auch verweigert werden. Kann aber tatsächlich eine staatliche Behörde die Zustimmung zur Kündigung einer Schwangeren bzw. eines Schwerbehinderten verweigern, wenn der 
Arbeitgeber infolge eines Wahlaktes in Fortfall geraten ist? Eine Institution, die durch das Wahlrecht und durch das Parlamentsrecht gelöscht wird, kann nicht durch das Sozialrecht als fortbestehend fingiert werden. Was wäre gewesen, wenn die Fraktion keine Rücklagen gebildet hätte?

Klagen, Klagen, Klagen

Infolge der Notwendigkeit, ordentliche Kündigungen auszusprechen, und infolge der dadurch bedingten Kosten der Lohnfortzahlung konnte die Fraktion in Liquidation die aus dem Sozialplan eingegangenen finanziellen Verpflichtungen wegen der Abfindungen nur zu einem Bruchteil erfüllen. Verhandlungen mit der Bundestagsverwaltung bzw. der Bundestagspräsidentin auf Bewilligung zusätzlicher Mittel blieben erfolglos. Ebenso blieben Verhandlungen erfolglos, die vorhandenen Mittel so umzuwidmen, daß sie für Fraktionsmitarbeiter und Abgeordnetenmitarbeiter gleichermaßen verwandt werden durften.

Bereits im Dezember 1990 reichte der Betriebsrat eine Klage gegen alle ehemaligen Abgeordneten der Bundestagsfraktion sowie gegen die Fraktion in Liquidation ein mit dem Ziel, feststellen zu' lassen, daß ehemalige Abgeordnete und Fraktion in Liquidation verpflichtet seien, den abgeschlossenen Sozialplan zu erfüllen. In Reaktion auf die Klage kündigte der Geschäftsführer der Fraktion in Liquidation an, er werde an die Abgeordneten die in den "Spendentopf « zugunsten der Abgeordnetenmitarbeiter eingezahlten Beträge rücküberweisen. Diese Ankündigung veranlaßte den Betriebsrat, gegen den Geschäftsführer eine einstweilige Verfügung zu erwirken, in der dem Geschäftsführer Auszahlungen aus dem "Spendentopf « untersagt wurden. Das Beschlußverfahren gegen die ehemaligen Abgeordneten und gegen die Fraktion in Liquidation führte vor dem Arbeitsgericht Bonn zu einem - vom Gericht vorgeschlagenen - Vergleich. Danach sollte der Sozialplan durch eine Einigungsstelle entsprechend den veränderten Bedingungen und unter Beachtung des Haushaltsrechts neu abgeschlossen werden und verpflichtete sich die Fraktion in Liquidation zu prüfen, ob zur Erfüllung des Sozialplanes Klagen gegen den Deutschen Bundestag erhoben werden können. Im Vergleich festgeschrieben wurde mithin die durch das Haushaltsrecht bewirkte unterschiedliche Behandlung zwischen Fraktionsmitarbeitern und Abgeordnetenmitarbeitern. Die ehemaligen Abgeordneten und die Fraktion in Liquidation wurden aus finanziellen Verpflichtungen für die Erfüllung des Sozialplans entlassen, soweit diese Verpflichtungen über die vorhandenen finanziellen Mittel hinausgingen, und es wurde eine Option auf die Zukunft durch Inaussichtstellen einer Klage vereinbart. In der mündlichen Verhandlung führte das Gericht zum Vergleichsvorschlag aus, daß eine Haftung der einzelnen Abgeordneten nicht in Betracht komme, weil es sich bei der Fraktion um einen nicht-rechtsfähigen Verein handele, auf den die Regeln des eingetragenen Vereins analog Anwendung fänden und bei dem nur das Vereinsvermögen haftet. ${ }^{12}$ Zum gleichen Ergebnis - eine individuelle Haftung käme nicht in Betracht - führte ein Antrag auf Prozeßkostenhilfe wegen einer in Aussicht genommenen Klage auf Abfindung eines früheren Abgeordnetenmitarbeiters gegen eine ehemalige Abgeordnete ${ }^{13}$.

Als Folge des arbeitsgerichtlichen Vergleiches zwischen Fraktion in Liquidation und Betriebsrat wurde gegen den Deutschen Bundestag eine Klage vor dem Verwaltungsgericht Köln eingereicht mit dem Ziel, den Bundestag zu verpflichten, Mittel bereitzustellen zur vollständigen finanziellen Erfüllung des Sozialplans. Die Klage wurde

12 Arbestsgericht Bonn, Protokoll der Sitzung vom 15.3. 1991 (5 BV 110/91).

13 Arbetsgericht Siegen, Beschluß vom 16.7.1991 (I Ca 222/91). 
als unzulässig abgewiesen mit dem Argument, es handele sich um einen Rechtsstreit verfassungsrechtlicher Art. Das argumentum e contrario, um einen solchen könne es sich nicht handeln, weil eine Fraktion in Liquidation jedenfalls nicht vor dem Bundesverfassungsgericht klagen könne und daher wegen der Rechtsschutzgarantie des Art. 19 Abs. 4 GG das Verwaltungsgericht zuständig sein müsse, wurde vom Verwaltungsgericht Köln nicht akzeptiert. ${ }^{14}$ Berufung und/oder Revision ist aus Kostengründen und deswegen nicht eingelegt worden, weil eine Entscheidung nicht vor r994 zu erwarten gewesen wäre und sie daher ihren sozialen Sinn verloren hätte.

Im Ergebnis ${ }^{14 a}$ veränderten die verschiedenen Klageverfahren nicht die Ausgangssituation: Die Fraktionsmitarbeiter und -mitarbeiterinnen erhielten Lohnfortzahlung während der Laufzeit ihrer Kündigungsfristen, sie wurden freigestellt und erhielten eine Abfindung, die etwa 20\% der im Sozialplan vereinbarten Abfindung betrug. Die Abgeordnetenmitarbeiter und -mitarbeiterinnen mußten sich infolge des auf das Ende der Legislaturperiode befristeten Arbeitsvertrages sofort arbeitslos melden. Sie kamen also nicht in den Genuß der bezahlten Freistellung, und die Abfindungsbeträge, die sie aus dem "Spendentopf « erhielten, waren kleiner.

\section{Sächliche Mittel}

In der ersten Besprechung mit der Bundestagsverwaltung über die Probleme der Liquidation der Fraktion hatte die Bundestagsverwaltung zu verstehen gegeben, sie halte die Fraktion für verpflichtet, die sächlichen Mittel, die aus den Fraktionszuschüssen erworben worden sind, an die Bundestagsverwaltung auszukehren ${ }^{15}$. Als diesem Argument mit der Erwägung begegnet wurde, daß - wenn eine Übergabe der sächlichen Mittel an die Bundestagsverwaltung erfolge - es auch konsequent sei, daß die Bundestagsverwaltung in die laufenden Arbeitsverträge eintrete, beharrte die Bundestagsverwaltung nicht mehr auf dem zuvor eingenommenen Rechtsstandpunkt. Nach Rücksprache mit dem Finanzministerium und dem Bundesrechnungshof wurde der Fraktion in Liquidation mitgeteilt, sie könne die sächlichen Mittel verwerten und die erhaltenen Mittel für die Finanzierung des Sozialplans einsetzen. So ist verfahren worden. Die sächlichen Mittel (PCs, Fahrzeuge, Drucker, Kopierer, Schreibmaschinen usw.) sind zu Höchstgeboten verkauft worden -- ein nicht ganz alltäglicher Fall für Gerätschaften, die zu 100\% aus Steuermitteln angeschafft worden sind.

\section{Kinderbaus}

Die Fraktion war Trägerin eines zu einem großen Teil aus Fraktionsmitteln finanzierten Betriebskindergartens. Mit Wegfall der Fraktion drohte die Schließung. Die Eltern gründeten daraufhin eine Elterninitiative, die Trägerin des Kindergartens wurde. Die Vermieterin des Anwesens, in dem der Kindergarten betrieben wurde, erklärte sich nach anfänglichen Bedenken wegen der finanziellen Leistungsfähigkeit

\footnotetext{
$1_{4}$ Verwaltungsgericht Koln, Urtell vom 5.3. 1992 (16 K 2655/91).

14 a Ein Verfahren eines Abgeordneten gegen den Deutschen Bundestag auf Feststellung, daß dieser verpflichtet sei, ihn von Verpflichtungen gegenuber seinen fruheren Mitarbeitern freizustellen, ist noch anhängig.

Is In diesem Sinne ist wohl der Kommentar von Ritzel/Bucker, Kommentar zur Geschaftsordnung des Bundestages, Anm. i zu $₫$ 10, zu verstehen. Der in den Verhandlungen anwesende Direktor beim Bundestag Bücker hat sich nicht geaußert. Kretschmer, (Fn. 4), S. 303, ist der Auffassung, die sảchlichen Gegenstande fallen beim Ende einer Fraktion ihrer Partei zu.
} 
bereit, die Eltern anstelle der Fraktion als Mieter zu akzeptieren. Die Fraktion in Liquidation gewährte der Elterninitiative ein finanzielles Darlehn zur Überbrükkung einer finanziellen Notlage. Das Darlehn wurde zurückgezahlt, nachdem der Elterninitiative Fördermittel des Landes zuflossen. Ferner wurden der Elterninitiative sächliche Mittel unentgeltlich übertragen, deren Verkehrswert in keinem Verhältnis zum Gebrauchswert stand. Dies betraf Kinderspielzeug und Einrichtungsgegenstände im Kinderhaus. Die Fraktion hat auf ihre Weise ermöglicht, daß das Kinderhaus fortexistieren konnte.

\section{Langfristige Verträge}

Die Fraktion hatte diverse Leih- und Lieferverträge mit Unternehmern vereinbart, deren Laufzeit weit über die Legislaturperiode gingen. Solche Verträge sahen regelmäßig Kündigungsfristen vor. Eine Vereinbarung, was zu geschehen hatte, wenn die Fraktion infolge des Wahlausgangs nicht mehr im Bundestag vertreten war, war nicht getroffen worden. Zivilrechtlich war zu entscheiden, ob der Grundsatz des "Wegfalls der Geschäftsgrundlage« zur Anwendung kommen konnte. Ein Großteil der Vertragspartner akzeptierte diesen Rechtsstandpunkt, und es kam zur einvernehmlichen Auflösung der Verträge. Ein kleinerer Teil akzeptierte diesen Rechtsstandpunkt nicht und drohte Klage an. Zu einem Verfahren ist es jedoch nicht gekommen. Angemahnt wurde die Gruppe BÜNDNIS 9o/GRÜNE, die zu Recht darauf hinwies, daß sie nicht Rechtsnachfolgerin der Fraktion DIE GRÜNEN/BÜNDNIS 90 war. Verklagt wurde auch die Partei DIE GRÜNEN, die ihrerseits geltend machen konnte, daß zwischen ihr und der Fraktion DIE GRÜNEN keine Identität bestand. Diejenigen, die wegen Nichteinhaltung von Kündigungsfristen gerichtlich gegen die Fraktion in Liquidation vorgehen wollten, verhedderten sich mithin im Bermuda-Dreieck zwischen Fraktion DIE GRÜNEN in Abwicklung, Gruppe BÜNDNIS 9o/GRÜNE und Partei DIE GRÜNEN.

\section{Akten}

Die Bundestagsfraktion DIE GRÜNEN/BÜNDNIS $9 \circ$ hatte Berge von Akten, Unterlagen, Korrespondenz usw. hinterlassen. Die Fraktion in Liquidation versuchte, dieser Hinterlassenschaft habhaft zu werden. Das war deswegen schwierig, weil im Dezember 1990 viele Abgeordnete und viele Mitarbeiter und Mitarbeiterinnen ihre Räume fluchtartig verlassen hatten. Die Hinterlassenschaft war deshalb ungeordnet und der Müll nicht von historisch wertvollem Material zu trennen. Eine rechtliche Verpflichtung zur Aufbewahrung von Fraktionsakten besteht lediglich wie bei anderen Unternehmen - für Personal- und Finanzakten. Für "politische» Akten gibt es keine rechtlichen Vorschriften. Das, was aus historischen Gründen sinnvoll ist, ist rechtlich ungeregelt. Die Gesamtheit der ungeordneten Akten wurde in Kisten verpackt und der Bundesgeschäftsstelle der Partei DIE GRÜNEN zur Aufbewahrung übergeben. Letztlich sind die Akten einem »Grünen Archiv« übertragen worden, dessen Träger der grünen-nahe Stiftungsverband Regenbogen ist. Die Finanz- und Personalakten stehen einstweilen der Fraktion in Liquidation zur Verfügung.

\section{Räume}

Anfang Januar 199r zog die Fraktion in Abwicklung auf Drängen der Bundestagsverwaltung aus ihren angestammten Räumen aus. Die Bundestagsverwaltung stellte 
für Zwecke der Abwicklung andere Räume zur Verfügung. Ab Sommer 199ı drängte die Bundestagsverwaltung darauf, die zur Verfügung gestellten Räumlichkeiten zurückzugeben. Dieses Ansinnen lehnte die Fraktion in Liquidation ab. Die Bundestagsverwaltung forderte nunmehr eine Nutzungsentschädigung. Auch dieser Forderung entsprach die Fraktion in Liquidation nicht. Parallel zur Forderung nach Nutzungsentschädigung klagte die Bundestagsverwaltung auf Herausgabe der Räume. Das Amtsgericht Bonn verwies die Klage mangels Zuständigkeit an das Verwaltungsgericht Köln. ${ }^{16}$ In der Erwägung der Dauer eines verwaltungsgerichtlichen Verfahrens entschloß sich die Bundestagsverwaltung zu einem Vergleich und stellte der Fraktion in Liquidation unbefristet und unentgeltlich zur Verwahrung der Personalund Finanzakten Kellerräume zur Verfügung.

\section{Rechtsnachfolge}

Alles wäre kein Problem gewesen, wenn die Gruppe BÜNDNIS 9o/GRÜNE Rechtsnachfolgerin der Fraktion DIE GRÜNEN/BÜNDNIS 90 gewesen wäre. Das ist sie jedoch nicht. Wegen des Grundsatzes der parlamentarischen Diskontinuität, der neu gewählte Abgeordnete davor schützt, in alte Hierarchien eingebunden zu werden, kann - auch das ist juristisch nicht unumstritten ${ }^{17}$ - eine Rechtsnachfolge nur entstehen, wenn ein entsprechender Beschluß der Abgeordneten vorliegt und im übrigen die Voraussetzungen des $\$ 6$ I 3 a BGB - Organisations- oder Teil-Organisationsnachfolge - vorliegen. Einen solchen Beschluß hat es nicht gegeben. Es gab auch keine Organisations- oder Teil-Organisationsnachfolge. Deswegen sind Arbeitsverhältnisse der Bundestagsfraktion DIE GRÜNEN/BÜNDNIS 90 nicht auf die Gruppe BÜNDNIS 9o/GRÜNE übergegangen. Selbstverständlich besteht auch nicht eine Rechtsnachfolge durch die Partei DIE GRÜNEN.

\section{Die Abricklung - Ein Ende ohne Ende?}

Im Frühsommer 1991 war ein Großteil der Abwicklung abgeschlossen. Eine Abwicklung ist jedoch ein zäher Vorgang. Im Sommer 1992 ist die Liquidation zu 99,5\% beendet. Bis auf Restmittel verfügt die Fraktion in Liquidation über keinerlei finanzielle Mittel mehr. Von 2,5 Mill. DM sind ca. 400000,-DM für Abfindungen, 2, I Mill. DM zur Begleichung von Verbindlichkeiten, insbesondere zur Zahlung von Löhnen, verwandt worden. Der "Spendentopf « in Höhe von $300000,-$ DM wurde zugunsten der Abgeordnetenmitarbeiter ausgeschüttet. Allerdings: Es gibt noch eine Besonderheit! Die Fraktion hatte Rücklagen für einen speziellen Zweck gebildet: Gegenüber Dienstherren von Beamten, die sich für die Tätigkeit bei der Fraktion hatten beurlauben lassen, mußte die Fraktion sich verpflichten, diese für die Zeit ihrer Tätigkeit bei der Fraktion und für den Fall nachzuversichern, daß sie endgültig aus dem Beamtenverhältnis ausscheiden. Hierfür ist eine Rücklage in Höhe von $200000,-$ DM gebildet worden. Da der jüngste beurlaubte Beamte, der bei der Fraktion als Angestellter tätig war, 199030 Jahre alt war, währt das Risiko bis zu dessen Pensionierung. Ob die Rücklagen die Risiken abdecken, läßt sich versicherungsmathematisch nicht feststellen. Es stellt sich daher die Frage, was passiert mit der Fraktion in Liquidation, wenn 1994 eine Bundestagsfraktion DIE GRÜNEN/BÜNDNIS 90 gebildet wird und diese Fraktion beschließt, wegen nicht kal- 
kulierbarer Risiken, nicht Rechtsnachfolgerin der Fraktion in Liquidation zu werden. In diesem Fall würde die Fraktion in Liquidation fortbestehen, und zwar solange, wie die sozialrechtlichen Risiken existieren. Was ist aber, wenn sich im Jahre 2025 erweist, daß Rücklagen übrig sind? Diese Frage werde ich erörtern, wenn ich im Jahre 2025 und im Alter von 78 Jahren genügend Zeit und Muße haben werde. Eine Veröffentlichung in der KJ ist für das Jahr 2026 mit der Redaktion abgesprochen.

\section{Hätte die Abwicklung anders verlaufen können?}

Politisch lag das Dilemma der Abwicklung darin begründet, daß sie sich reduzierte auf eine Auseinandersetzung zwischen Abgeordneten und Geschäftsführer einerseits und Mitarbeitern, insbesondere Abgeordnetenmitarbeitern, andererseits. Ein finanziell anderes Ergebnis, z. B. durch Nachbewilligung von Mitteln durch den Deutschen Bundestag, hätte sich - wenn überhaupt - nur erzielen lassen, wenn Abgeordnete und Mitarbeiter gemeinsam an die Öffentlichkeit gegangen wären mit dem Ziel, andere Regelungen und eine Nachbewilligung zu erreichen. Ein solches gemeinsames Vorgehen wäre auch politisch Signal für einen Neuanfang gewesen. Zu dieser, aus politischen und finanziellen Gründen notwendigen Gemeinsamkeit waren alle Beteiligten nicht mehr fähig. Wären sie dazu fähig gewesen, wäre wohl auch der Einzug einer neuen Fraktion in den Deutschen Bundestag nicht verfehlt worden.

Es kam, wie es kommen mußte: Abgeordnete, die einem Sozialplan zustimmten, dessen finanzielle Seite nur durch das Prinzip Hoffnung gedeckt war, mußten in Konflikt geraten mit Mitarbeitern und Mitarbeiterinnen, die einer auf Sand gebauten Regelung vertrauten. Was fraktionsintern vielen als alternatives Recht erschien, erwies sich bloß als Nicht-Recht.

\section{Hätte die Abwicklung anders verlaufen müssen?}

Unter rechtsstaatlichen Gesichtspunkten ist das Fehlen von gesetzlichen Regelungen, die dann zur Geltung kommen, wenn eine Fraktion nicht den Einzug in den Bundestag bzw. in Landtage schafft, ein weiteres dunkles - wenngleich kleineres Kapitel der im weitesten Sinne verstandenen Parteienfinanzierung: Zur Abwicklung sind überwiegend öffentliche Mittel verwandt worden. Die Verteilung dieser Mittel erfolgte nach bestem Wissen und Gewissen. Ich denke, Steuerzahler und Steuerzahlerinnen haben Anspruch auf etwas anderes, und zwar darauf, daß Steuermittel nach Kriterien verteilt werden, die der Gesetzgeber vorgibt. Eine solche gesetzliche Vorgabe hätte die Abwicklung beschleunigt, finanziell billiger gemacht und im übrigen dem Autor dieses Beitrages ein paar graue Haare erspart. 